\title{
Transcriptome Sequencing Revealed an Inhibitory Mechanism of Aspergillus flavus Asexual Development and Aflatoxin Metabolism by Soy-Fermenting Non-Aflatoxigenic Aspergillus
}

\author{
Kunlong Yang ${ }^{1,2,+} \mathbb{0}$, Qingru Geng ${ }^{1,+}$, Fengqin Song ${ }^{1,+}$, Xiaona He ${ }^{1}$, Tianran $\mathrm{Hu}^{2}$, \\ Shihua Wang ${ }^{2, *}$ and Jun Tian ${ }^{1, *(1)}$ \\ 1 School of Life Science, Jiangsu Normal University, Xuzhou 221116, China; ykl_long@yeah.net (K.Y.); \\ qingru950504@163.com (Q.G.); songfengqin@jsnu.edu.cn (F.S.); xiaona_he@163.com (X.H.) \\ 2 Key Laboratory of Pathogenic Fungi and Mycotoxins of Fujian Province, School of Life Sciences, \\ Fujian Agriculture and Forestry University, Fuzhou 350002, China; hutianran77@yeah.net \\ * Correspondence: wshmail@m.fafu.edu.cn (S.W.); tj-085@jsnu.edu.cn (J.T.); Tel.: +86-516-83403172 (J.T.) \\ + These authors contributed equally to this work.
}

Received: 29 July 2020; Accepted: 17 September 2020; Published: 23 September 2020

\begin{abstract}
Aflatoxins (AFs) have always been regarded as the most effective carcinogens, posing a great threat to agriculture, food safety, and human health. Aspergillus flavus is the major producer of aflatoxin contamination in crops. The prevention and control of A. flavus and aflatoxin continues to be a global problem. In this study, we demonstrated that the cell-free culture filtrate of Aspergillus oryzae and a non-aflatoxigenic $A$. flavus can effectively inhibit the production of AFB1 and the growth and reproduction of $A$. flavus, indicating that both of the non-aflatoxigenic Aspergillus strains secrete inhibitory compounds. Further transcriptome sequencing was performed to analyze the inhibitory mechanism of $A$. flavus treated with fermenting cultures, and the results revealed that genes involved in the AF biosynthesis pathway and other biosynthetic gene clusters were significantly downregulated, which might be caused by the reduced expression of specific regulators, such as AflS, FarB, and MtfA. The WGCNA results further revealed that genes involved in the TCA cycle and glycolysis were potentially involved in aflatoxin biosynthesis. Our comparative transcriptomics also revealed that two conidia transcriptional factors, $b r l A$ and $a b a A$, were found to be significantly downregulated, which might lead to the downregulation of conidiation-specific genes, such as the conidial hydrophobins genes $\operatorname{rod} A$ and $\operatorname{rodB}$. In summary, our research provides new insights for the molecular mechanism of controlling AF synthesis to control the proliferation of A. flavus and AF pollution.
\end{abstract}

Keywords: Aspergillus flavus; transcriptome; aflatoxin; conidiation; RNA-seq

\section{Introduction}

Aspergillus flavus is a common saprophytic fungus that contaminates many important seed crops, including peanuts, corn, and pistachios [1,2]. The contamination of A. flavus causes huge economic losses to agricultural production across the world. This fungus is also an opportunistic pathogen for immunocompromised patients, which is the second most common cause of aspergillosis after A. fumigatus [3]. A. flavus is notorious for its production of one of the most carcinogenic mycotoxins, aflatoxin [4], which has an extensive relationship with liver cancer [5]. Therefore, it is important to develop effective and safe approaches to control this fungus and inhibit the production of aflatoxins for food safety and human health. 
To prevent the contamination of A. flavus and aflatoxins on agricultural products and foodstuffs, which occur mostly at pre- and post-harvest periods, farmers and manufacturers have kept them at conditions of low temperature and low humidity. Numerous strategies, including physical control (e.g., heat and ultraviolet radiation), chemical control (e.g., using natural preservative) [6-8], and biological control (e.g., microbial competition), have been applied to combat this fungus and control aflatoxin production. Due to the efficient elimination of mycotoxins and to the desire for a safe food supply, biological control represents an attractive choice. A range of microbes, such as bacteria [9-11], yeast, and fungi (e.g., nontoxigenic Aspergillus), have been used for biocontrol aflatoxigenic Aspergillus [12]. Several biological control strategies to reduce AF contamination have been developed, including the use of non-toxic $A$. flavus and other fungi to inhibit aflatoxin synthesis [13]. Shakeel et al. reported that the bacterium Streptomyces yanglinensis 3-10 was able to produce antifungal substances that reduce the postharvest decay of peanut kernels by inhibiting mycelia growth of A. flavus and $\mathrm{AFB}_{1}$ production [14]. A previous study reported that spreading non-toxic A. parasiticus strains on peanut-growing soil could reduce the AF content in edible peanuts by $83 \%$ to $98 \%$ [15]. Fungi, such as white-rot fungus, Rhizopus pseudomonas, and A. niger, have been utilized to control aflatoxin biosynthesis [16]. Of these filamentous fungi, non-toxigenic aspergilli used for fermented foods, such as A. oryzae and A. niger, could be the best prospect for the sake of their safety.

Transcriptome sequencing has been utilized for a better understanding of the inhibitory mechanisms of antagonistic microbes against $A$. flavus. A biocontrol yeast against $A$. flavus called Wickerhamomyces anomalus was found to inhibit AF production by repressing the activation of the AF biosynthetic pathway cluster [17]. The extracts from the medicinal plant Micromeria graeca were also reported to restrict AF biosynthesis, without an effect on fungal growth, by the downregulation of aflR and aflS, two activators of the AF gene cluster, and the overexpression of two major global regulators, $v e A$ and $m t f A$ [18]. Zhao et al. previously revealed the inhibitory effects of Lactobacillus plantarum on AF biosynthesis using transcriptomic analysis, which showed that the transcriptional levels of genes involved in the AF biosynthetic process were significantly downregulated, while genes related to the synthesis and organization of cell wall polysaccharides were upregulated, which might be related to the impaired effects of L. plantarum on the cellular structure of fungal tissue [19].

In this study, we used two non-aflatoxigenic Aspergillus strains (A. oryzae and an MAPK kinase SskB null mutant of $A$. flavus named TSJ-1 that fails to produce aflatoxins) to control the growth and development of the aflatoxin synthesis of $A$. flavus. We found that, in a co-culture system with $A$. oryzae and a non-aflatoxigenic A. flavus generated genetically, the production of AFB1 was dramatically inhibited. Furthermore, the cell-free culture filtrate of these two non-aflatoxigenic Aspergillus strains was enabled and effectively blocked AF biosynthesis and A. flavus development as well. Further comparative transcriptome sequencing was applied to reveal the inhibition mechanism of AF metabolism and A. flavus development by A. oryzae.

\section{Results}

\subsection{The Effect of Non-Aflatoxigenic Aspergilli on A. flavus Growth and Aflatoxin B1 (AFB1) Production by Co-Cultivation with A. flavus}

To test whether the non-aflatoxigenic Aspergillus strains affect A. flavus growth and AFB1 accumulation under a co-culture system, a total number of $10^{6}$ conidia of $A$. oryzae RIB40/TSJ-1 were co-cultivated with an equal amount of $A$. flavus NRRL3357 spores in $50 \mathrm{~mL}$ of YES medium for 9 days. The results showed that the total biomass of the co-culture system displayed no difference compared to the wild type (Figure 1B), while A. flavus co-cultivated with A. oryzae was greater in mycelium pellets compared with the wild-type control, and smaller ones were found when co-cultured with TSJ-1 (Figure 1A). The experiment that $A$. flavus co-cultivated with different concentrations of spores of $A$. oryzae/TSJ-1 demonstrated that an increasing conidia amount to $10^{6}$ of the non-aflatoxigenic Aspergillus strains could apparently block AFB1 accumulation (Figure 1C). The AFB1 production in the co-culture medium with an equal amount of Aspergillus spores $\left(10^{6}\right)$ was assayed after 3 days and 
after 9 days of incubation as well, which showed that AFB1 production was decreased after 9 days of incubation in the wild-type control compared to the 3 days of incubation (Figure 1D), and AFB1 production was dramatically inhibited in the co-culture system at both of these two time points, while a detectable level of AFB1 could still be found in the TSJ-1 co-cultures (Figure 1D).

A

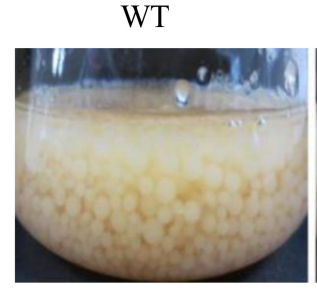

$\mathrm{C}$

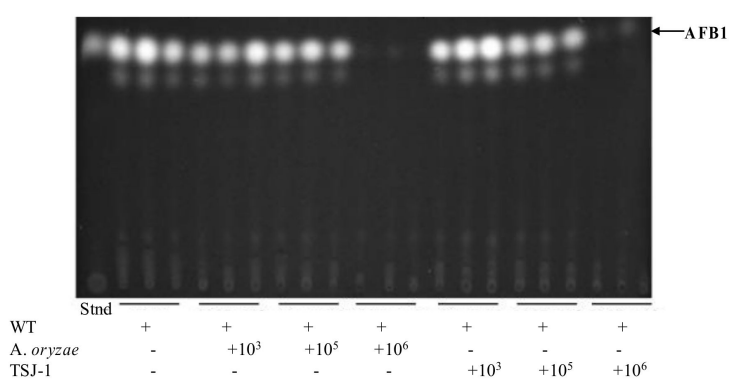

WT+TSJ-1

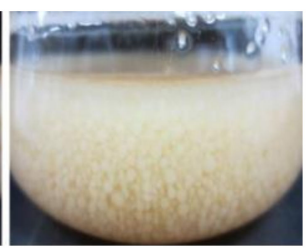

B

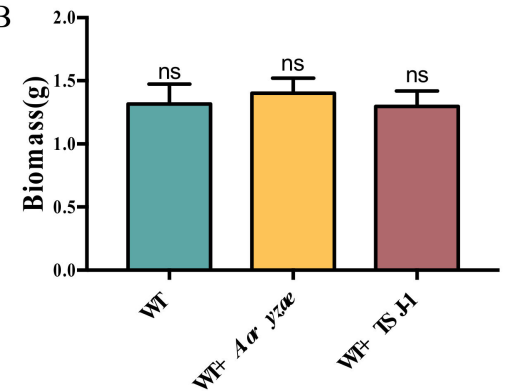

$\mathrm{D}$

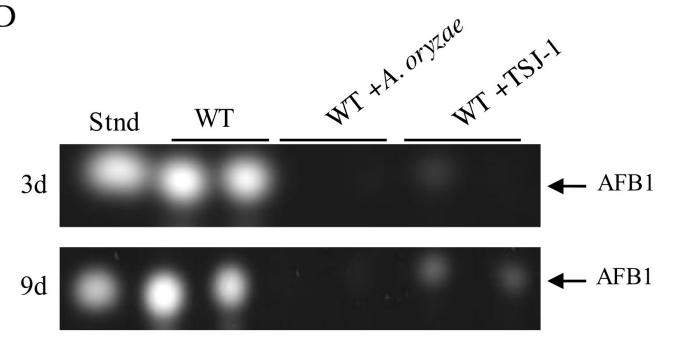

Figure 1. The growth phenotype and aflatoxin B1 production of $A$. flavus when co-cultured with non-aflatoxigenic Aspergillus strains. (A) The growth phenotype of A. flavus when co-cultured with A. oryzae or TSJ-1 strain in liquid-shake YES (yeast extract and sucrose) medium at $30^{\circ} \mathrm{C}$ for 9 days. (B) The total mycelium dry weight of $A$. flavus co-cultured with the indicated strains. ns indicates no significance. (C) Detection result of aflatoxins production after $A$. flavus was co-cultured with different concentrations of non-aflatoxigenic Aspergillus strains' spores at $30^{\circ} \mathrm{C}$ for 4 days. In total, $1 \mathrm{~mL}$ of $10^{6}$ A. flavus spores was added to a 50-mL YES medium. (D) TLC results of AFB1 production when A. flavus was co-cultured with the indicated strains in a liquid-shake YES medium for 3 and 9 days, respectively.

2.2. Cell-Free Concentrated Filtrates of Non-Aflatoxigenic Aspergilli inhibit A. flavus Asexual Development and AFB1 Accumulation

To determine the effect of the culture filtrate of A. oryza and TSJ-1 on A. flavus development, A. flavus was inoculated on solidified PDA plates containing with or without different concentrations of the concentrated filtrates. An inhibitory growth of wild-type A. flavus was found within the treatment of $A$. oryzae culture filtrates and $8 \%$ of the TSJ- 1 culture filtrate (Figure $2 \mathrm{~A}, \mathrm{~B}$ ). The result also showed that a significant decrease in conidia pigmentation occurred in the treatment of $A$. oryzae/TSJ-1 culture filtrates when compared to the wild-type control (Figure 2A). Further determination of conidiation showed that $A$. flavus was significantly reduced in conidia production in the treatment of TSJ-1 culture filtrates and $8 \%$ of $A$. oryzae culture filtrate (Figure $2 \mathrm{C}$ ). 
A

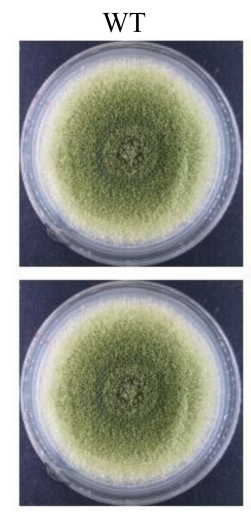

WT

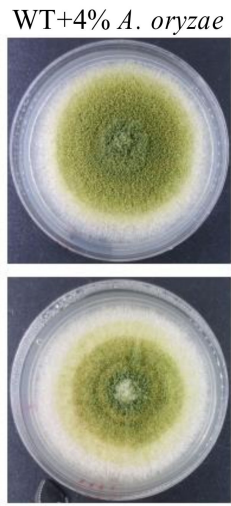

$\mathrm{WT}+4 \% \Delta T S J-1$

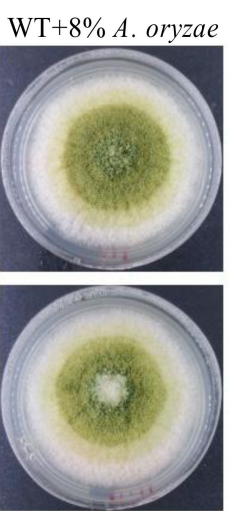

$\mathrm{WT}+8 \% \mathrm{TSJ}-1$
B

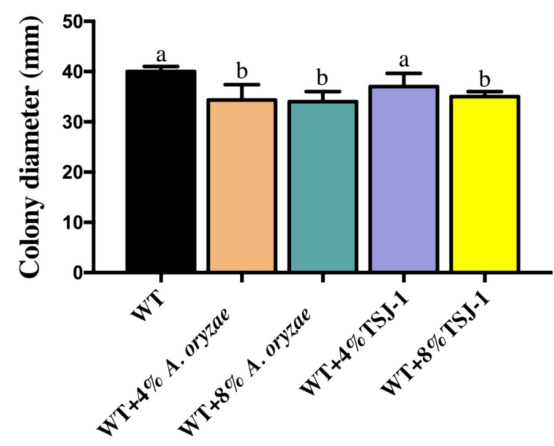

$\mathrm{D}$

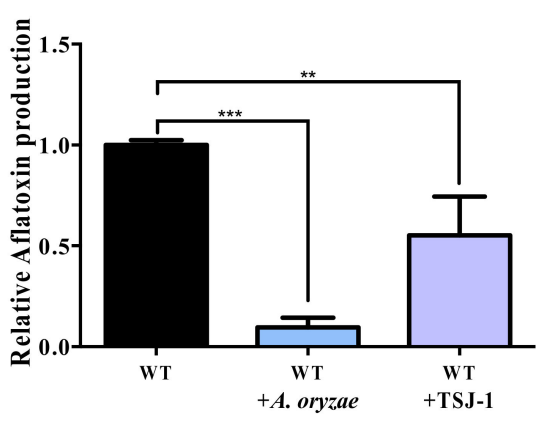

Figure 2. Effect of the culture filtrate of non-aflatoxigenic Aspergillus strains on A. flavus reproduction and AFB1 production. (A) Growth phenotype of the wild-type A. flavus strain on PDA medium treated with different concentrations of the culture filtrate of the indicated strains for 4 days. (B) Colony diameter of A. flavus in (A). (C) Conidia production of A. flavus in (A). (D) The effect of the culture filtrate of the indicated stains on AFB1 production in a liquid-shake YES medium for 4 days. A total of $10^{6}$ A. flavus spores were added to a 50-mL YES medium with $8 \%$ of the culture filtrate of the indicated strains. Different uppercase letters above the bars represent significantly different values $(p<0.01)$, while different lowercase letters above the bars represent $p<0.05$. ${ }^{* *}$ statistically significant when compared to control, $p<0.01 ;{ }^{* * *}$ statistically significant when compared to control, $p<0.001$.

To assay its influence on AFB1 biosynthesis, A. flavus NRRL3357 was grown in YES media supplemented with $8 \%$ of the concentrated filtrates. The results showed that $A$. oryzae culture filtrates were found to be highly effective in inhibiting AFB1 production with an inhibition rate of 90.43\%, while the inhibition rate for the TSJ-1 filtrates was $44.8 \%$ (Figure 2D). Taken together, these data demonstrated that filtrates of non-aflatoxigenic aspergilli have inhibitory effects on $A$. flavus asexual conidiation and AFB1 production.

\subsection{RNA-seq Analysis of A. flavus by the Treatment of A. oryzae Culture Filtrate}

To reveal the regulatory molecular mechanism of non-aflatoxigenic aspergilli culture filtrate against $A$. flavus, RNA-seq analysis was carried out. Here, since the A. oryzae RIB40 WT strain was found more effective in inhibiting AFB1 production, A. flavus vegetatively grown in the presence or absence of $A$. oryzae culture filtrate was further sampled and analyzed using RNA-seq. A total of 38.52 $\mathrm{Gb}$ of clean base of $6 \mathrm{cDNA}$ libraries were gained, and more than $5.7 \mathrm{~Gb}$ clean base for each biological repeat were obtained (Table S1). The Q20 and Q30 for each biological repeat were over 90\% and 96\%, respectively (Table S1). RNA sequences of A. flavus NRRL 3357 were further processed by quantile normalization of counts per million of counts uniquely mapping to each gene model, and only unique reads were used for the calculation of normalized gene expression as RPKM (reads 
per kilobase of transcript per million mapped reads). To characterize gene sets in response to the treatment of $A$. oryzae filtrates, a Venn diagram (Figure $3 \mathrm{~A}$ ) and volcano plots (Figure 3B) according to $\log 10$ of padj ( $y$-axis) and $\log 2$ of fold change ( $x$-axis) were used to visualize the common differentially expressed genes (DEGs), which indicated that more than 3100 DEGs (including 1204 downregulated and 1929 upregulated genes) were found in response to the treatment of $A$. oryzae filtrates (Figure 3A, Table S3). Analysis of the top 10 upregulated/downregulated DEGs showed that a gene predicted to encode a hypothetical FAD/NAD(P)-binding protein (AFLA_124990), a putative cytochrome P450 oxidoreductase GliC-like gene (AFLA_023030), and the fucose-specific lectin gene fleA (AFLA_065960) was one of the most highly expressed genes, increased by more than 800-fold compared with control A. flavus, while the conidial hydrophobin gene rodA (AFLA_098380), a putative spherulin 4-like cell surface protein coding gene (AFLA_002020), and a putative efflux pump antibiotic resistance protein coding gene (AFLA_125070) were one of the most downregulated genes, decreased by more than 50-fold compared to the wild-type control (Figure 3C). Additionally, we found that most of the molecular chaperone (heat shock proteins) and two alternative oxidases were transcriptionally activated in response to the treatment of $A$. oryzae filtrates (Figure S1B), and, intriguingly, most of the $\mathrm{G}$ protein-coupled receptors were found to be downregulated (Figure S1A).

A

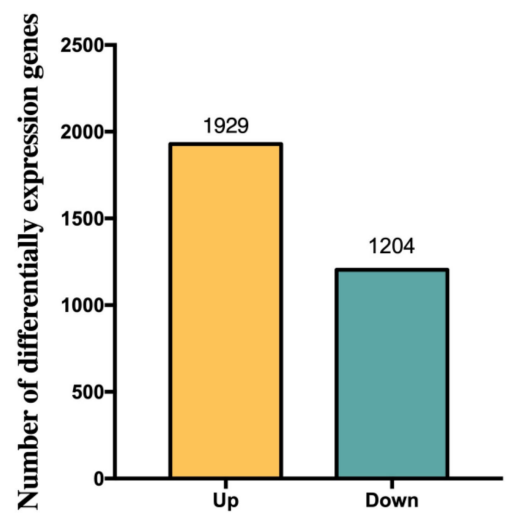

B

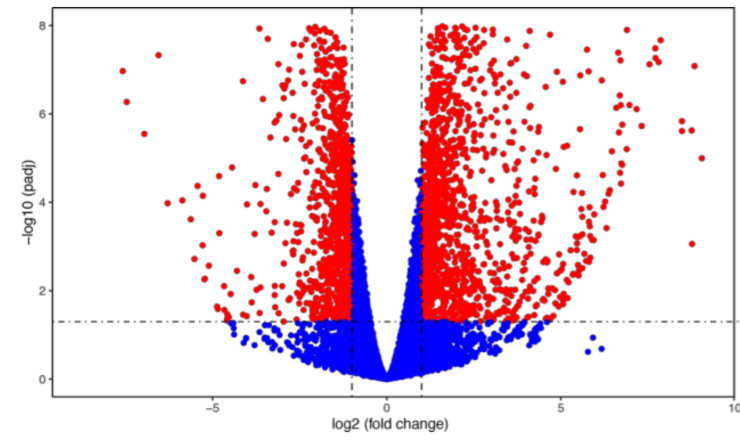

$\mathrm{C}$

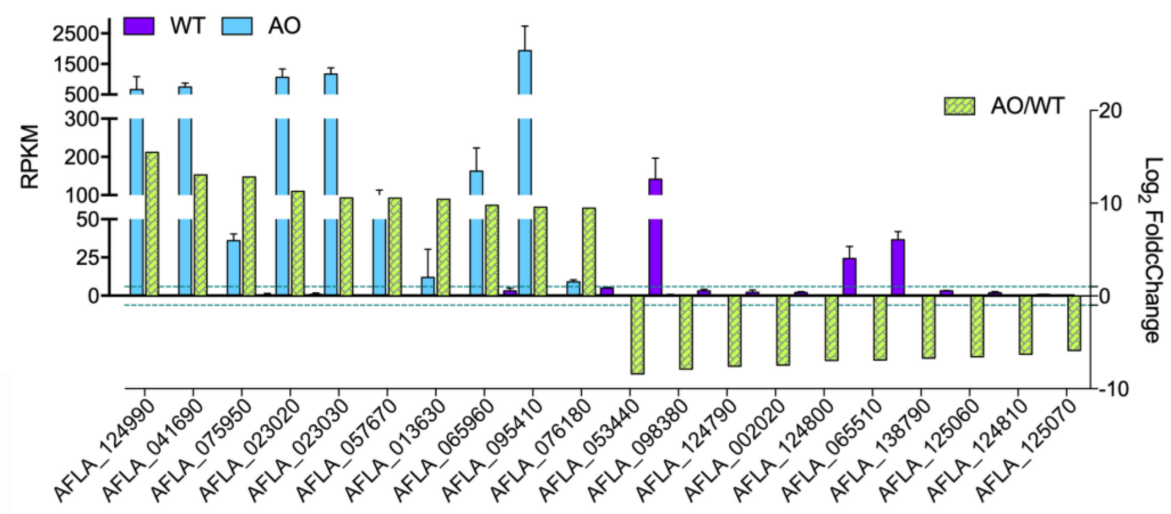

Figure 3. Analysis of differentially expressed genes (DEGs) in A. flavus treatment with A. oryzae Rib40 cell-free culture filtrate. (A) Number of genes showing upregulated and downregulated expression of WT strain cultured in PDB with/without $A$. oryzae cell-free culture filtrate treatment. (B) Volcano plots of the DEGs in the wild-type strain with or without $A$. oryzae culture filtrate treatment. The blue spots inside the grey dash lines indicate the non-significant DEGs. (C) Relative expression levels of the top 10 upregulated/downregulated DEGs. Left $Y$ axis indicates the RPKM values of the selected DEGs in WT (A. flavus wild type without any treatment) and AO (A. flavus wild type with A. oryzae cell-free culture filtrate treatment); Right $Y$ axis presents the $\log 2$ FoldChange of the selected DEGs in AO when compared to WT. The dashed line indicates $\mid \log 2$ FoldChange $\mid=1$. 


\subsection{GO Enrichment and KEGG Pathways Analysis of DEGs}

The DEGs were further utilized for GO term analysis, including biological processes, cellular components, and molecular functions. The oxidation-reduction process (GO:0055114), carbohydrate catabolic process (GO:0016052), and transmembrane transport (GO:0055085) were the most significantly enriched GO terms in biological process, while the integral component of the membrane (GO:0016021)/extracellular region (GO:0005576) and the oxidoreductase activity (GO:0016491)/catalytic activity (GO:0003824)/binding (GO:0031177, GO:0019842, GO:0072341) were the most enriched GO terms in cellular component and molecular function (Figure 4A), respectively. Functional enrichment of the KEGG pathway of the upregulated and downregulated DEGs was also characterized. Enrichment analyses of the upregulated DGEs in the treatment of $A$. oryzae filtrates demonstrated that $\mathrm{ABC}$ transporters, genes involved in the primary metabolism (such as carbon metabolism and amino acid metabolism) and the secondary metabolism, were significantly enriched (Figure 4B). Additionally, the downregulated DGEs were significantly enriched in the metabolic pathway, antibiotic biosynthesis, and the precursors of secondary metabolites, such as terpenoid backbone and one-carbon pool (Figure 4C), indicating that the synthesis of secondary metabolites in A. flavus might be inhibited with the treatment of A. oryzae filtrates (Figure 4C).

A
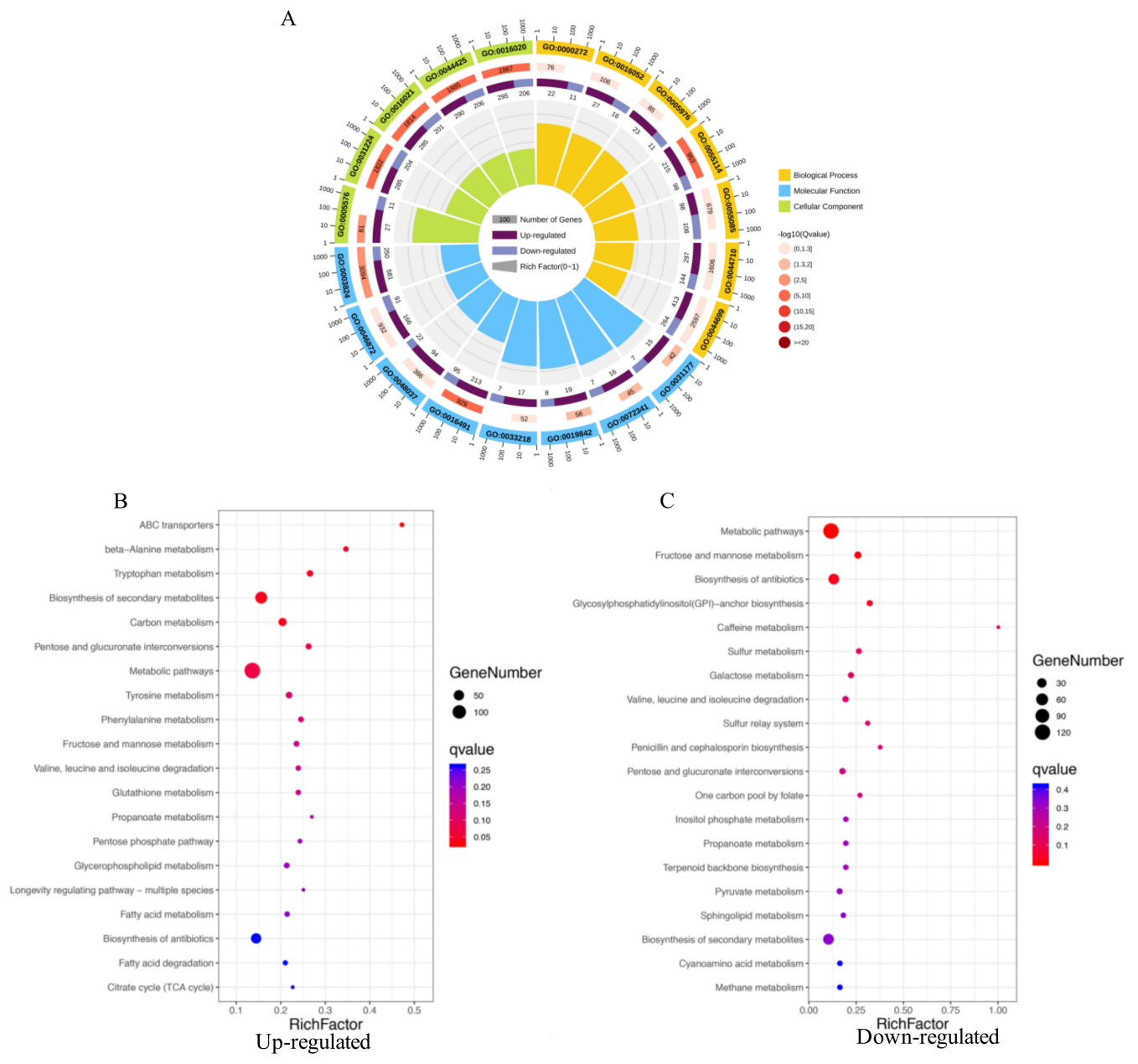

Figure 4. Enriched GO terms and KEGG pathways of differentially expressed genes (DEGs). (A) Enriched GO terms of DEGs. The numbers on the periphery represent a ruler for gene numbers, while the numbers in boxes indicate the threshold value of the "-log qvalue". (B) Enriched KEGG pathways of the 1929 upregulated DEGs and (C) the 1204 downregulated DEGs that show the top 20 pathways of KEGG enrichment analysis. 


\subsection{Inhibition of Aflatoxin Biosynthesis Gene Cluster by the Treatment of A. oryzae Filtrates}

To better understand how A. oryzae filtrates affect AF biosynthesis in A. flavus, the expression levels of 29 genes that were required for the generation of $\mathrm{AF}$ within the biosynthesis gene cluster were observed and compared. The enzymatic reactions of AF are involved in three stages: early, middle, and late stages (Figure 5A). Here, we found that genes involved in early and middle stages of enzymatic reactions of AF were significantly downregulated in their transcription levels with the treatment of $A$. oryzae filtrates compared to those enzymes functioning at the late stage (Figure $5 \mathrm{~B}$ ). Interestingly, despite the pathway-specific regulatory transcription factor, AflR did not show a difference at its transcription expression level, and its partner, AflS, was transcriptionally inhibited under the treatment of $A$. oryzae filtrates (Figure 5B). In A. flavus, dozens of regulators have been reported to be participating in the regulation of AF production [20]. The transcriptome analysis demonstrated that a negative regulator of $\mathrm{AF}, \mathrm{NsdC}$ [21], was activated under the treatment of $A$. oryzae filtrates (Figure 5C), while many of the positive regulators of AF, such as FarB [22], MtfA [23], and StuA [20], were significantly decreased in their expression levels (Figure 5C).

A

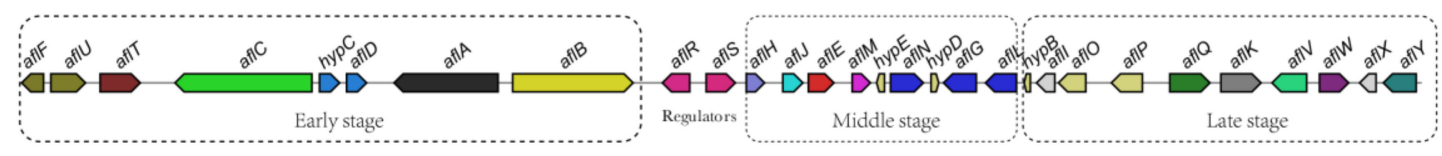

B

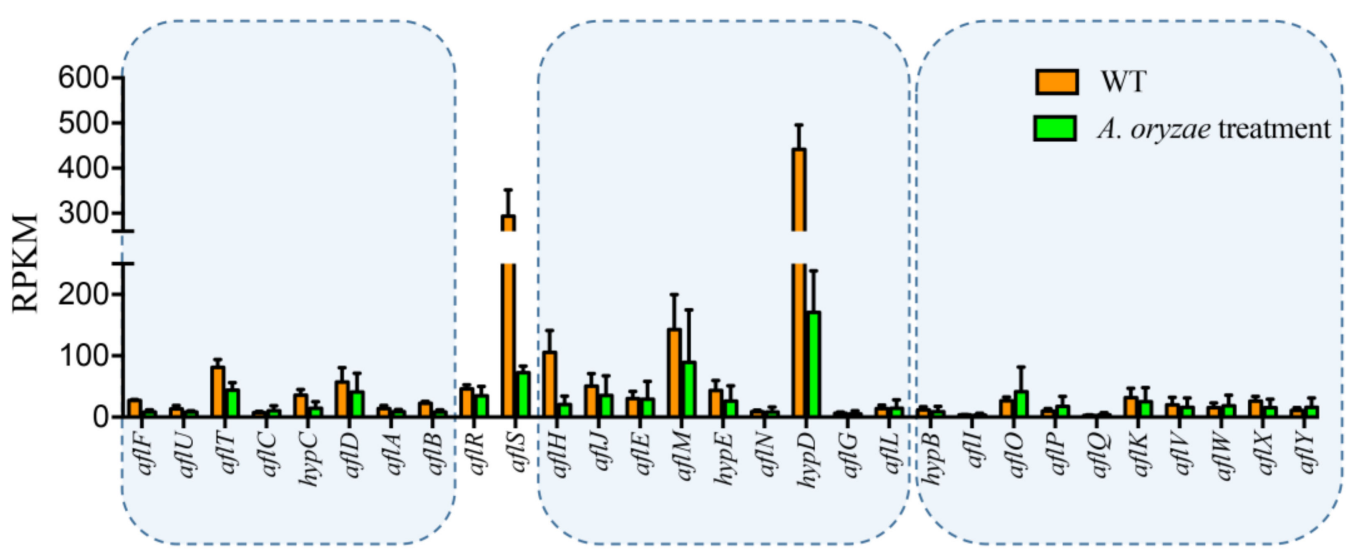

C

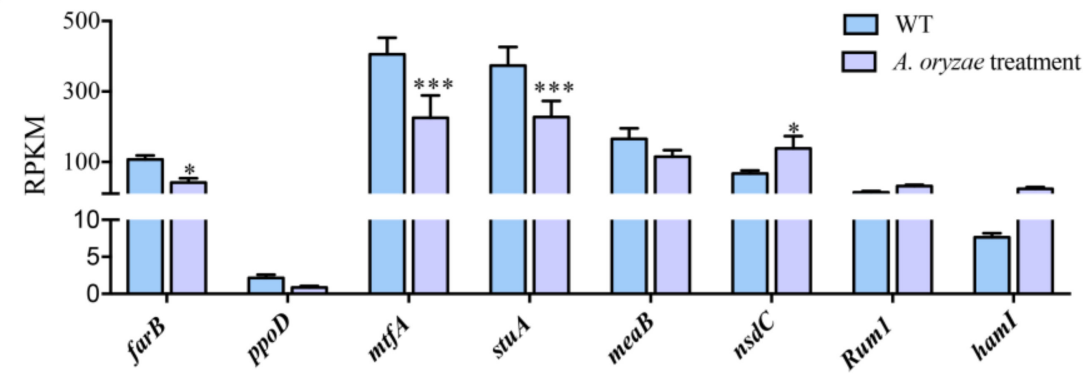

Figure 5. Effects of cell-free culture filtrate of A. oryzae Rib40 on the transcriptional expression of different stages of aflatoxin genes. (A) Aflatoxin gene cluster in A. flavus that was grouped into three stages according to the intermediates produced by their proteins in aflatoxin biosynthesis. (B) Expression levels (RPKM) of the 29 aflatoxin biosynthesis-related genes with/without $A$. oryzae Rib40 cell-free culture filtrate treatment. (C) Expression levels of known aflatoxin regulators. ${ }^{*}$ statistically significant when compared to control, $p<0.05 ;{ }^{* *}$ statistically significant when compared to control, $p<0.001$. 


\subsection{The Effect of A. oryzae Filtrates on the Expression of Biosynthetic Gene Clusters (BGCs)}

The enrichment analysis demonstrated that DGEs involved in the metabolic pathway and the precursors of secondary metabolites were significantly downregulated in their expression (Figure 4). To determine whether A. oryzae filtrates have an impact on the other BGCs, we first analyzed the expression levels of 13 transcriptional factors (TFs) located in the predicted BGCs (Figure 6A). Former studies have revealed 74 BGCs, including the experimental identified SM in A. flavus [24,25], and here we found that only 13 transcriptional factors were located in the 11 predicted BGCs (Table S2), among which the expression levels of genes AFLA_128160 encoding a TF of cluster 5, AFLA_096330 and AFLA_096370 encoding two TFs of cluster 31 (expressing Aflatrem), and AFLA_059960 encoding a TF of cluster 71 were downregulated by more than twofold (Figure 6A). We further analyzed the expression data of the BGCs that are identified experimentally to produce SMs in A. flauvus. The results showed that most of the genes in the clusters of aflavarin and aspterric acid, together with aflatoxin localized in chromosome III, were transcriptionally reduced in their expressions. Most genes that are involved in the biosynthesis of leporin B, clavaric acid, and aflatrem were found to be depressed as well, while genes involved in the production of cyclopiazonic acid, imizoquin, and PR-toxin were significantly activated in response to A. oryzae filtrates (Figure 6B).

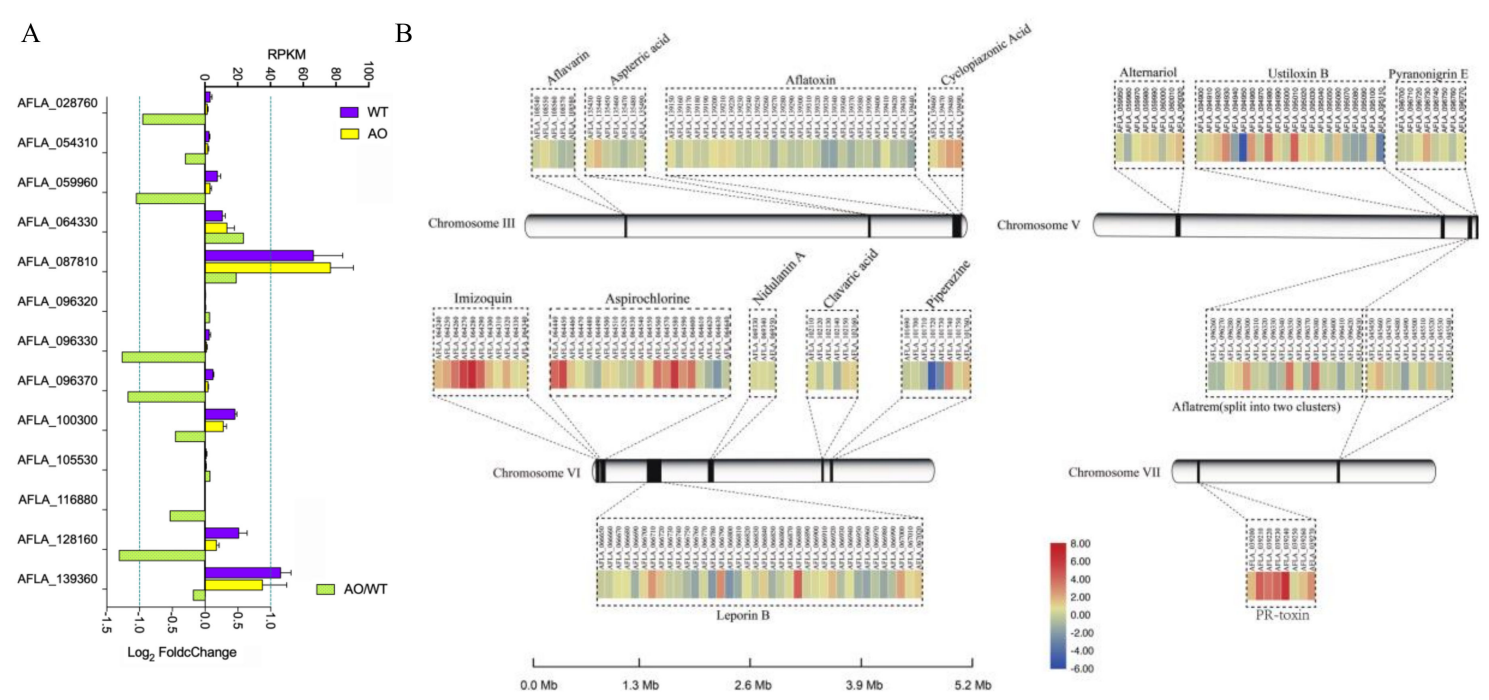

Figure 6. Effects of cell-free culture filtrate of A. oryzae Rib40 on gene expression of the biosynthesis gene clusters (BGCs) that are identified experimentally in A. flavus. (A) Relative expression levels of 13 transcriptional factors encoding genes identified among the BGCs in A. flavus. Top X axis indicates the RPKM values of the selected DEGs in WT (A. flavus wild type without any treatment) and AO (A. flavus wild type with $A$. oryzae cell-free culture filtrate treatment); bottom $X$ axis presents the $\log 2$ FoldChange of the selected DEGs in AO when compared to WT. The dashed line indicates $\mid \log 2$ FoldChange $\mid=1$. (B) Heat map and localization of experimentally identified BGCs in A. flavus. Heat map and chromosomal position of BGCs were visualized with TBtools [26]. To make the heat map, the original PRKM data were used and normalized with a log scale. A newly published A. flavus genome was used as a reference to visualize the chromosomal position of the BGCs [27].

To better understand how A. oryzae filtrates affected the expression of BGCs transcriptionally, the expression levels of more than $200 \mathrm{TFs}$ reported in A. flavus were analyzed. A total of $66 \mathrm{TFs}$ were found to be expressed differently by more than twofold, among which half of them ( 33 for each) were significantly upregulated and downregulated, respectively (Figure 7A). To determine whether these TFs share a similarity in their expressing pattern, a correlation heat map was analyzed, and the result showed that AF-specific TF AflR was negatively correlated with AFLA_097920 (a putative C6 transcription factor). Intriguingly, another AF regulator AflS showed an expressed correlation 
with farB2 (a C6 transcription factor), AFLA_083560 (a putative C6 transcription factor), brlA, abaA, and AFLA_084200 (a putative C6 transcription factor) (Figure 7B).

A

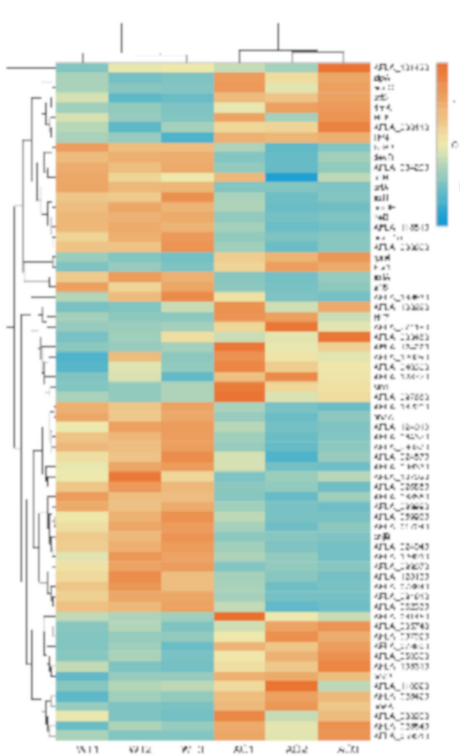

B

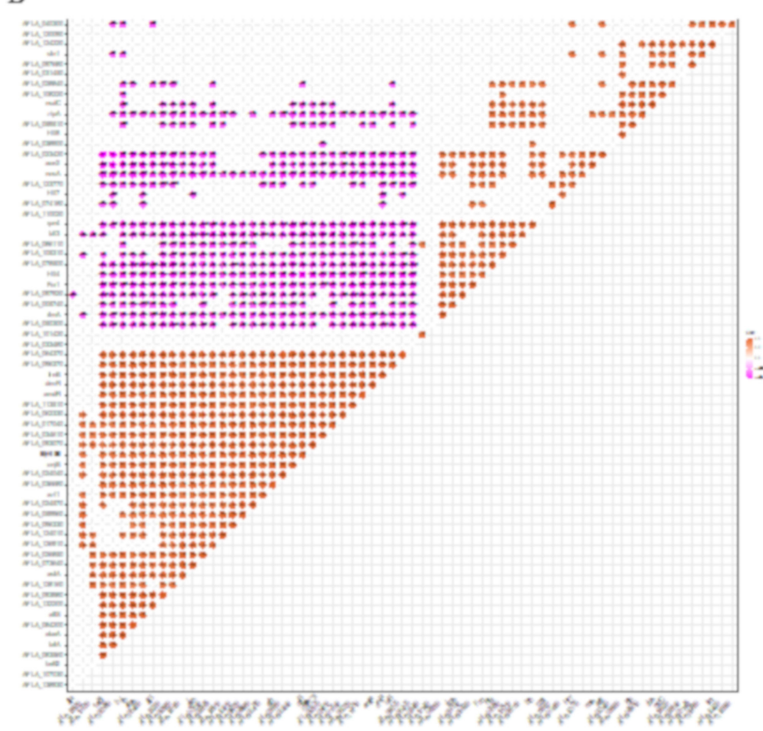

Figure 7. Effects of cell-free culture filtrate of A. oryzae Rib40 on the expression of the transcriptional factors encoding genes in A. flavus. (A) Clustering/heat map visualization of differentially expressed genes that encode transcriptional factors in A. flavus. (B) Correlation heat map of the DEGs that encode transcriptional factors in A. flavus. Data with a $p$ value $>0.05$ were excluded from the figure.

\subsection{Co-Regulated Gene Expression Network between Aflatoxigenic and Non-Aflatoxigenic Conditions}

Aflatoxins have been shown to regulate many environmental factors (such as $\mathrm{pH}$ and temperature) and culture conditions [28]. We previously reported that A. flavus failed to produce AF grown in YEP media [20]. To explore the general regulation mechanism of aflatoxins in A. flavus, an interaction network analysis between the aflatoxigenic and non-aflatoxigenic conditions ( $A$. oryzae filtrate treatment and YEP-cultured media) was analyzed by WGCNA. The results demonstrated that the indicated RNA-seq data of YEP and with A. oryzae filtrate treatment (AO) or without A. oryzae filtrate treatment (CK) were clustered into 20 modules, which were marked with different colors (Figure 8A). The MEturquoise and MEbrown modules displayed the highest correction with the aflatoxin phenotype $\left(\mathrm{R}^{2}=0.95\right.$ and $\mathrm{R}^{2}=0.96$, respectively). A total of 325 interacted genes of the MEturquoise module were utilized to generate the network (Figure 8B), while 87 genes (591 in total) of the MEbrown module were interacted to generate the co-expression network (Figure $8 \mathrm{C}$ ). The information of the predicted interaction network is indicated in Table S4. The connection between the CADAFLAP gene number, from the String online program, and AFLA gene number is indicated in Table S5. Here, we found that three AF-related genes, including AFLA_139330 (aflH, CADAFLAP00007809), AFLA_139370 (aflB, CADAFLAP00007812), and AFLA_139400 (hypC, CADAFLAP00007815), which were all downregulated in the non-aflatoxigenic conditions, were identified in the MEturquoise module gene list. In the MEturquoise module, phosphoglycerate kinase PgkA (AFLA_069370, CADAFLAP00008361), GMP synthase (AFLA_137950, CADAFLAP00007672), pyruvate kinase (AFLA_087900, CADAFLAP00001568), nitrate reductase NiaD (AFLA_018810, CADAFLAP00003766), Cu,_Zn superoxide dismutase SOD1 (AFLA_099000, CADAFLAP00011638), and a ubiquitin-like modifier SUMO (AFLA_068730, CADAFLAP00008297) were among the core regulated network, which were all downregulated both in the A. oryzae filtrate treatment and YEP-cultured media (Figure 8B). Intriguingly, the most connected proteins, including citrate synthase Cit1 (AFLA_007020, CADAFLAP00010765), fumarate hydratase (AFLA_091270, CADAFLAP00008812), fructose-1,6-bisphosphatase Fbp1 (AFLA_027310, CADAFLAP00002106), and isocitrate lyase AcuD (AFLA_052400, CADAFLAP00011231) were identified among the MEbrown 
module gene list (Figure 8C). Importantly, the expression levels of their encoding genes, which are involved in the TCA (tricarboxylic acid) cycle and glycolysis, were all significantly reduced in the non-aflatoxigenic conditions. It was valuable to notice that the TCA cycle and glycolysis were potentially involved in aflatoxin biosynthesis here.

A

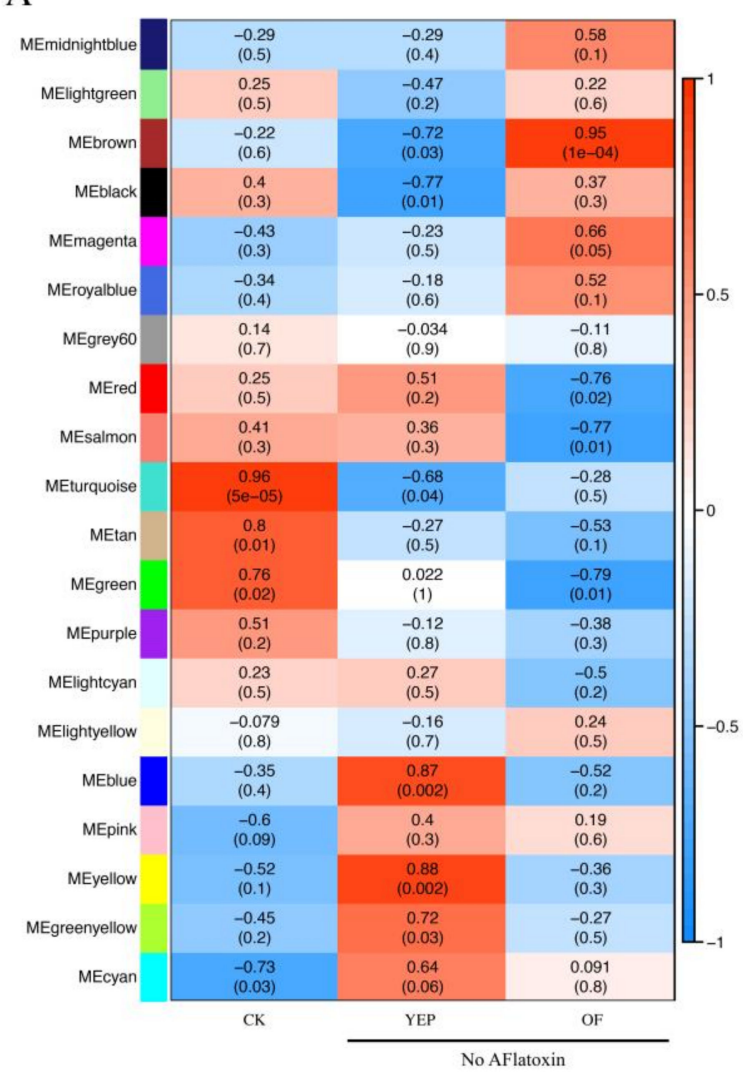

B

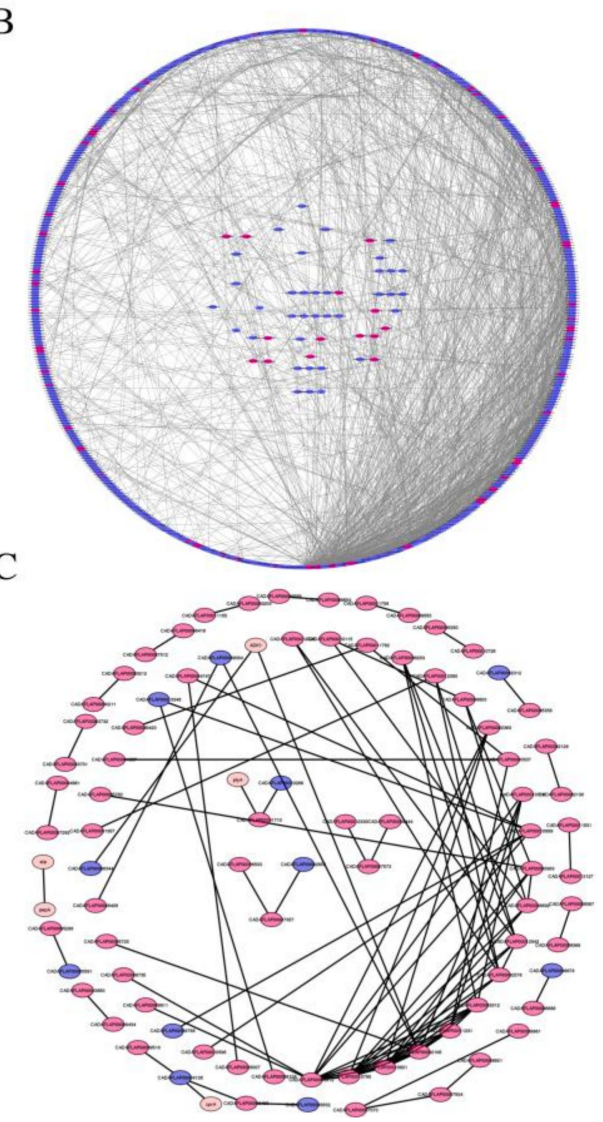

Figure 8. Analysis of the gene co-expression network between aflatoxigenic and non-aflatoxigenic conditions. (A) Association of the aflatoxin phenotype and consensus module eigengenes was performed with WGCNA analysis in aflatoxigenic and non-aflatoxigenic conditions. Gene network of the MEturquoise (B) and MEbrown (C) module was analyzed with the String online program (https://string-db.org) and further visualized with Cytoscape. Genes that were upregulated in a non-aflatoxigenic condition are indicated in red, while downregulated genes in a non-aflatoxigenic condition are shown in blue. CK, A. flavus wild type grown on GMM medium without any treatment; YEP, A. flavus wild type grown on an aflatoxin non-conducing YEP medium; AO, A. flavus wild type grown on a GMM medium with $A$. oryzae filtrate treatment. Values in (A) represent a positive correlation unless preceded by a minus, in which case values represent a negative correlation. The values in brackets indicate the $p$ value.

\subsection{Inhibitory Regulation of Asexual Development Genes by the Treatment of A. oryzae Filtrates}

Conidiation is one of the most important reproductive structures of A. flavus that can help it to spread in the environment and cause a series of contamination by this fungus. In this study, we found that $A$. oryzae filtrates could suppress asexual development of A. flavus (Figure 2). To determine the inhibitory regulation of $A$. oryzae filtrates on $A$. flavus reproduction, the transcriptional conditions of genes involved in asexual development were analyzed (Figure 9). Two important transcriptional factors that regulate asexual development, $\mathrm{BrlA}$ and $\mathrm{AbaA}$, were found significantly decreased in their expression (Figure 9A,B), which might cause the downregulation of conidiation-specific genes, 
such as the conidial hydrophobins genes $\operatorname{rod} A$ and $\operatorname{rodB}$ (Figure 9A). In the regulatory networks, negative regulators of conidiation, such as Nsdc, MedA, PhnA, RlmA, and, in the velvet protein complex, VeA, were found remarkably increased in their expression at the transcriptional level in response to the A. oryzae filtrates (Figure 9B). Genes related to signal transduction, such as RAS small monomeric GTPase ras $A$, Rheb small monomeric GTPase coding gene $r h b A$, and MAP kinase coding gene $m p k B$, which have been reported to be involved in asexual development, were transcriptionally downregulated (Figure 9B). Taken together, the transcriptional data demonstrated the negative effects on asexual reproduction involved in the regulation of positive and negative regulators of conidiation in A. flavus.

A

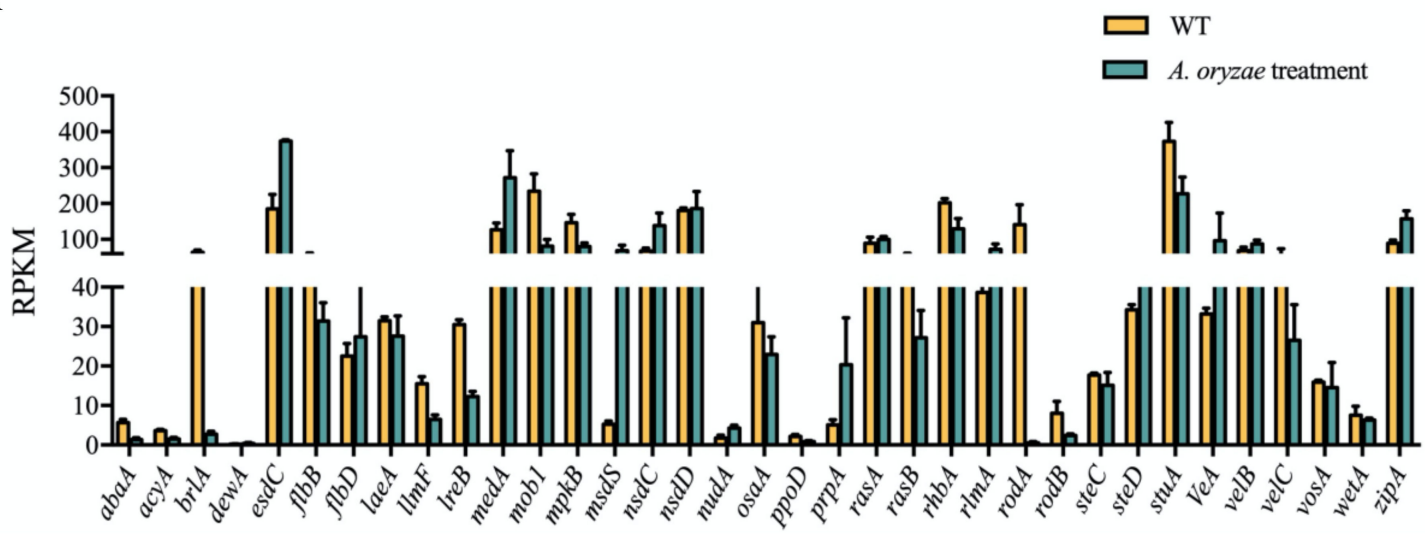

B

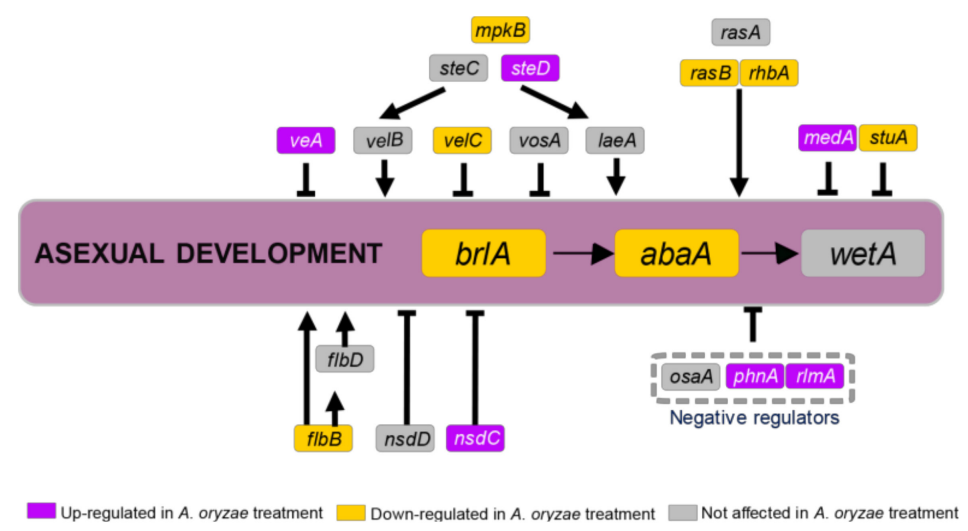

Figure 9. Effects of cell-free culture filtrate of A. oryzae Rib40 on the expression of genes that are involved in asexual development in A. flavus. (A) Expression levels (RPKM) of genes that are involved in asexual development with/without $A$. oryzae Rib40 cell-free culture filtrate treatment. (B) A schematic diagram of the regulatory model of asexual development. Genes with increased, decreased, and unaffected mRNA levels in the A. oryzae Rib40 cell-free culture filtrate are labeled in red, green, and gray, respectively.

\section{Discussion}

A. flavus is a saprophytic soil fungus that is notorious for its ability to colonize pre-harvest and post-harvest seed crops with one of the most toxic secondary metabolite aflatoxins, which has caused billion-dollar yield losses across the world [29]. This fungus is also an opportunistic pathogen of human and animals, causing aspergillosis diseases mostly due to the dispersion of the asexual spores in the air. This fungus is also hard to eliminate, both as a plant and human pathogen, due to its resistance to many common fungicides and a limited ability to apply fungicides to edible portions of plants or foodstuffs [30]. However, several different methods have been developed to combat this fungus and decrease the losses caused by its contamination and by mycotoxins [2,31-33]. 
Several studies indicating that the interaction of A. niger and other aspergilli with A. flavus is able to block aflatoxin biosynthesis have been reported [34-36]. In this study, we utilized A. oryzae and a non-aflatoxigenic $A$. flavus generated genetically to co-culture with wild-type $A$. flavus, and found that the production of AFB1 was remarkably inhibited (Figure 1). The cell-free culture filtrate of A. oryzae could more effectively inhibit the production of AFB1, which is consistent with the former study [34]. Early studies on A. niger demonstrated that, in a co-culture system with A. flavus, A. niger produced oxalic acid to suppress aflatoxin biosynthesis, partially due to the decrease in substrate $\mathrm{pH}$ levels of below 3.0 [37]. The antagonistic microbes against $A$. flavus, such as Bacillus megaterium, Penicillium chrysogenum, and $A$. niger, have been shown to secrete small antifungal peptides with low molecular weight that could inhibit AFB1 biosynthesis [38-40]. A similar result was found in this study. The cell-free culture filtrate was collected using a $1 \mathrm{KDa}$ dialysis system to force metabolites and media through the filter. The inhibitory impact of the concentrated filtrates on A. flavus reproduction and AFB1 production suggested a presence of signal molecules in the culture filtrate that were able to restrict $A$. flavus development and AFB1 biosynthesis (Figure 2). Further research on the identification of the signal molecules produced by the non-aflatoxigenic aspergilli is needed to elucidate these findings further.

Nevertheless, the mechanism of the inhibition of AFB1 biosynthesis by these aspergilli remains unclear. In this study, to analyze the inhibitory mechanism of A. flavus treated with fermenting cultures, transcriptome sequencing was performed, which demonstrated that genes involved in the early and middle stages of enzymatic reactions of AFB1 were significantly decreased in their expression levels with the treatment of $A$. oryzae filtrates (Figure 5). One of the AF pathway-specific regulator AflS was found to be transcriptionally downregulated in response to the treatment of $A$. oryzae filtrates (Figure 5). Some other transcription factors (TFs) that have been reported to be involved in the regulation of AF production were found to be expressed differently in this study. For instance, a negative regulator of $\mathrm{AF}, \mathrm{NsdC}$, was found to be upregulated under the treatment of $A$. oryzae filtrates (Figure 5), while the positive regulators of AF, such as FarB, MtfA, and StuA, were significantly decreased in their expression levels (Figure 5). The differential expression of these TFs could potentially inhibit the activation of the BGC of AFB1, leading to a reduction of AF biosynthesis. In addition to AFB1, the A. oryzae filtrates were found to have a similar inhibitory impact on many of the biosynthesis gene clusters in A. flavus. Most genes that are involved in biosynthesis of aflavarin, leporin B, and aflatrem were found to be significantly downregulated (Figure 6), while genes involved in cyclopiazonic acid, imizoquin, and PR toxins were significantly activated in response to A. oryzae filtrates (Figure 6). In $A$. nidulans, the antifungal protein PAF isolated from $P$. chrysogenumare was shown to inhibit the growth of $A$. nidulans by interfering with PCK/MPK and cAMP/PKA signals [41]. Here, we also found that most of the heat shock proteins were transcriptionally activated (Figure S1), while most of the $\mathrm{G}$ protein-coupled receptors were found to be downregulated in response to the treatment of $A$. oryzae filtrates (Figure S1), which might lead to an inactivation of their downstream signaling pathway. Intriguingly, our WGCNA results revealed that genes involved in the TCA cycle and glycolysis were significantly downregulated in the non-aflatoxigenic conditions (Figure 8C). It has been demonstrated that, in addition to providing energy, the TCA cycle and glycolysis are the major sources of precursors of secondary metabolites in filamentous fungi [29]. Thus, blocking the TCA cycle and glycolysis can potentially inhibit biosynthesis of many important secondary metabolites, including aflatoxins.

The inhibition of the secreted proteins produced by aspergilli on A. flavus growth has been reported in many studies [35,42]. Here, we found that the asexual development of $A$. flavus was obviously suppressed by A. oryzae filtrates (Figure 2). In Aspergillus molds, asexual development is regulated by the BrlA $>$ AbaA $>$ WetA transcriptional cascade [24]. Our comparative transcriptomics revealed that brlA and $a b a A$ (but not wet $A$ ) were found to be significantly decreased in their expression (Figure 9), while some other negative regulators of conidiation, such as velvet protein complex, VeA, Nsdc, MedA, PhnA, and RlmA, were transcriptionally activated in response to the A. oryzae filtrates (Figure 9), which might together lead to the downregulation of conidiation-specific genes, such as the conidial 
hydrophobins genes $\operatorname{rod} A$ and $\operatorname{rodB}$ (Figure 9). Intriguingly, the $\mathrm{C} 2 \mathrm{H} 2$-type conidiation transcription factor BrlA that is required for asexual development in Aspergillus molds was found to be a key regulator of BGCs and secondary metabolites that are regulated by LaeA in an epigenetic manner in A. fumigatus [43]. Although laeA expressed no difference in response to the A. oryzae filtrate treatment, brlA was found to be remarkably downregulated, which might partially explain why many of the BGCs were suppressed in response to A. oryzae filtrate treatment (Figure 6).

\section{Materials and Methods}

\subsection{Strains and Culture Conditions}

The Aspergillus strains used in this study are indicated in Table 1, among which A. flavus NRRL 3357 is an aflatoxigenic wild-type stain, A. oryzae RIB40, and TSJ-1 (an MAPK kinase SskB-null mutant of $A$. flavus that fails to produce aflatoxins) are served as non-aflatoxigenic aspergilli. All strains were inoculated on potato dextrose agar (PDA) medium (20\% potato, $2 \%$ dextrose, and $1.5 \%$ agar) and cultured in the dark at $30^{\circ} \mathrm{C}$ for 7 days. Spores were collected from individual cultures on PDA with 0.001\% Tween-20 solution after filtering mycelia with four layers of wipe paper. The spores were quantified hemocytometrically, and the numbers were adjusted to $10^{6}$ conidia/mL with distilled water for further study.

Table 1. Aspergillus strains used in this study.

\begin{tabular}{ccc}
\hline Name of Strain & Genotype & Source \\
\hline NRRL 3357 & A. flavus Wild type & Keller lab [3] \\
RIB40 & A. oryzae Wild type & Keller lab [3] \\
TSJ-1 & $\Delta k u 70, \Delta s s k B:$ AfupyrG & Wang lab \\
\hline
\end{tabular}

4.2. Effect of Co-Cultivation of Non-Aflatoxigenic Aspergilli on the Growth of A. flavus and AFB1 Production

A series of dilution of non-aflatoxigenic Aspergillus strains' spores $\left(10^{3}, 10^{5}\right.$, and $10^{6}$ spores $\left./ \mathrm{mL}\right)$ were, respectively, added to a 50-mL YES (yeast extract and sucrose) medium containing $1 \mathrm{~mL}$ of $1 \times 10^{6}$ conidia/mL suspensions of $A$. flavus NRRL3357, and incubated in the dark at $30{ }^{\circ} \mathrm{C}$ for 9 days with shaking at $150 \mathrm{rpm}$. The control without additional spores of non-aflatoxigenic Aspergillus was performed under the same conditions. The experiments were conducted with three replicates for all treatments. The AFB1 production in the culture medium was assayed after 3 days and after 9 days of incubation. Aflatoxins were then extracted according to a previously described method [44]. Briefly, $500 \mu \mathrm{L}$ of culture medium were used for AF extraction with chloroform and thin-layer chromatography (TLC) was performed to analyze AFB1 production.

\subsection{Effect of the Culture Filtrate of Non-Aflatoxigenic Aspergilli Strains on A. flavus Development and AFB1 Production}

The analysis of the cell-free culture filtrate of non-aflatoxigenic aspergilli on wild-type $A$. flavus development and AF biosynthesis was performed according to the previously reported method with minor modification [35]. A total number of $10^{7}$ A. oryzae/TSJ-1 conidia were added to $500 \mathrm{~mL}$ of potato dextrose broth (PDB) and cultured at $30{ }^{\circ} \mathrm{C}$ for 5 days with shaking at $180 \mathrm{rpm}$.

The cell-free culture filtrate was collected by filtering hyphae. To concentrate the culture filtrates, a 1 KDa dialysis bag (Sangon Biotech, Shanghai, China) with the filtrates was placed over a bed of polyethylene glycol (PEG) and covered with more PEG, and the compounds with a weight less than $1 \mathrm{KDa}$ were dialyzed from the bag. The concentrated culture filtrates were filtered with a 0.2- $\mu \mathrm{m}$-diameter filter for future use. To assay the effect of concentrated cell-free culture filtrates of non-aflatoxigenic aspergilli on $A$. flavus development, $2 \mu \mathrm{L}$ of $10^{6}$ conidia/mL of wild-type $A$. flavus were spotted onto solidified PDA plates containing $4 \%$ or $8 \%$ of the concentrated filtrates, and were incubated for 7 days at $30{ }^{\circ} \mathrm{C}$ in the darkness. The PDA plates without supplemental concentrated 
filtrates were used as a control. The diameter of the colony and the spore's production of A. flavus were measured after 7 days of inoculation.

All treatments were tested in three replicates. To assay its influence on AFB1 biosynthesis, $1 \mathrm{~mL}$ of $1 \times 10^{6}$ conidia/mL suspensions of $A$. flavus NRRL3357 was added to $50 \mathrm{~mL}$ of YES with a supplementation of $8 \%$ of the concentrated filtrates, and were incubated in the dark at $30^{\circ} \mathrm{C}$ for 4 days with shaking at $150 \mathrm{rpm}$. The extraction and detection of AFB1 are described above. For quantitative analysis of AF production from the TLC result, the ImageJ software was used.

\subsection{RNA Isolation}

A total number of $10^{7}$ A. flavus wild-type conidia were added to $50 \mathrm{~mL}$ of glucose minimal medium (GMM) and cultured at $30^{\circ} \mathrm{C}$ for $24 \mathrm{~h}$ with shaking at $180 \mathrm{rpm}$, and $8 \%$ of the concentrated filtrates of $A$. oryzae RIB40 were then added to the medium with continued culturing at $30{ }^{\circ} \mathrm{C}$ for $24 \mathrm{~h}$. The experiments were conducted with three replicates. The mycelia were harvested and frozen in liquid nitrogen, and were lyophilized for $24 \mathrm{~h}$. Total RNA was extracted with Trizol (Invitrogen, Carlsbad, CA, USA), according to the manufacturer's protocol. The quality and integrity of RNA samples were determined using a Nanodrop and an Agilent 2100 bioanalyzer (Agilent Technologies, Palo Alto, CA, USA), respectively. The quantity of RNA samples was further measured with a Qubit RNA assay kit (Life Invitrogen, Carlsbad, CA, USA).

\subsection{RNA-Seq and Enrichment Analysis of Differentially Expressed Genes}

The total RNA of three biological replicates for the treatment of $A$. oryzae concentrated filtrates and control (without treatment) was sequenced. A standard protocol from Illumina Inc. (San Diego, CA, USA) and sequencing on a HiSeq 2000 platform (Berry Genomics, Beijing, China) were used for preparing the libraries. The sequenced clean reads were mapped against predicted transcripts of the A. flavus NRRL 3357 genome using hisat2 [45] and Samtools [46].

Transcript abundance was estimated using the Featurecount package [47]. Differentially expressed genes (DEGs) were screened by a comparison of $A$. oryzae concentrated filtrate treatment groups with control groups and then analyzed with DESeq package using RStudio software, and both a 2-fold change cut-off and an adjusted $p$-value of $\leq 0.05$ were established as thresholds. The enriched GO terms and KEGG pathways of DEGs were analyzed with OmicShare Tools (https://www.omicshare.com/tools/).

\subsection{Weighted Correlation Network Analysis (WGCNA) of Co-Expression Gene Network}

The co-expression gene network was analyzed by a WGCNA R package [48]. The RPKM (reads per kilobase per million mapped reads) of all A. flavus genes were used as input for the WGCNA, and an "unsigned" type was applied to create the network. The weighted matrix of pair-wise connection strengths (module) was built, and genes were grouped into modules by hierarchical clustering. The power $\beta$ with a value of 9 was used to calculate the correlation coefficients. The selective eigengenes module was analyzed with the String online program (https://string-db.org) and further visualized with Cytoscape.

\subsection{Statistical Analysis}

The results were analyzed using one-way analysis of variance (ANOVA) and Student's $t$-test as appropriate with GraphPad Prism 7 software (San Diego, CA, USA) for significance analysis of multiple comparisons and comparison of two different groups, respectively. Each treatment consisted of three replicates and was expressed as mean $\pm \mathrm{SD}$ (standard deviation).

\section{Conclusions}

Our data showed that A. oryzae and the non-aflatoxigenic Aspergillus have potential biocontrol activity to inhibit aflatoxin biosynthesis and $A$. flavus asexual development. Comparative transcriptomics 
further revealed the inhibitory mechanism of aflatoxin metabolism and asexual development in A. flavus. This study may potentially provide the antifungal agent against $A$. flavus and aflatoxins, which are safe for agricultural harvest.

Supplementary Materials: Supplementary materials can be found at http://www.mdpi.com/1422-0067/21/19/ 6994/s1.

Author Contributions: K.Y., J.T., and S.W. designed the experiments and wrote the manuscript. K.Y., Q.G., and F.S. performed all the experiments. X.H., and T.H. performed a few experiments and data analysis. All authors have read and agreed to the published version of the manuscript.

Funding: This research was supported by the National Natural Science Foundation of China (31900036, 31972171, 31671944), the Natural Science Foundation of Jiangsu Province (BK20190994), Six Talent Peaks Project of Jiangsu Province (SWYY-026), Qing Lan Project of Jiangsu Province, the Program of Natural Science Foundation of the Jiangsu Higher Education Institutions of China (19KJB180016), the Program of Natural Science Foundation of Jiangsu Normal University (18XLRX029), the China Scholarship Council, Postgraduate Research \& Practice Innovation Program of Jiangsu Province (KYCX20_2300) and the Priority Academic Program Development of Jiangsu Higher Education Institutions (PAPD).

Acknowledgments: The authors would like to thank Nancy P. Keller from University of Wisconsin, Madison, for providing suggestions and assistance for this manuscript, and thank Shimuye Kalayu Yirga from Fujian Medical University for the help of language editing of this manuscript.

Conflicts of Interest: The authors declare that there are no conflicts of interest.

\section{Abbreviations}

$\begin{array}{ll}\text { AF } & \text { Aflatoxin } \\ \text { DEGs } & \text { differentially expressed genes } \\ \text { RPKM } & \text { Reads Per Kilobase per Million mapped reads } \\ \text { GO } & \text { Gene Ontology } \\ \text { KEGG } & \text { Kyoto Encyclopedia of Genes and Genomes } \\ \text { WGCNA } & \text { Weighted correlation network analysis }\end{array}$

\section{References}

1. Diener, U.L.; Cole, R.J.; Sanders, T.; Payne, G.A.; Lee, L.S.; Klich, M.A. Epidemiology of aflatoxin formation by Aspergillus flavus. Annu. Rev. Phytopathol. 1987, 25, 249-270. [CrossRef]

2. Qu, S.; Yang, K.; Chen, L.; Liu, M.; Geng, Q.; He, X.; Li, Y.; Liu, Y.; Tian, J. Cinnamaldehyde, a promising natural preservative against Aspergillus flavus. Front. Microbiol. 2019, 10, 2895. [CrossRef] [PubMed]

3. Yang, K.; Shadkchan, Y.; Tannous, J.; Landero Figueroa, J.A.; Wiemann, P.; Osherov, N.; Wang, S.; Keller, N.P. Contribution of ATPase copper transporters in animal but not plant virulence of the crossover pathogen Aspergillus flavus. Virulence 2018, 9, 1273-1286. [CrossRef] [PubMed]

4. Xing, F.; Ding, N.; Liu, X.; Selvaraj, J.N.; Wang, L.; Zhou, L.; Zhao, Y.; Wang, Y.; Liu, Y. Variation in fungal microbiome (mycobiome) and aflatoxins during simulated storage of in-shell peanuts and peanut kernels. Sci. Rep. 2016, 6, 25930. [CrossRef] [PubMed]

5. Marchese, S.; Polo, A.; Ariano, A.; Velotto, S.; Costantini, S.; Severino, L. Aflatoxin B1 and M1: Biological properties and their involvement in cancer development. Toxins 2018, 10, 214. [CrossRef]

6. Tian, J.; Gan, Y.; Pan, C.; Zhang, M.; Wang, X.; Tang, X.; Peng, X. Nerol-induced apoptosis associated with the generation of $\mathrm{ROS}$ and $\mathrm{Ca}(2+)$ overload in saprotrophic fungus Aspergillus flavus. Appl. Microbiol. Biotechnol. 2018, 102, 6659-6672. [CrossRef]

7. Song, F.; Geng, Q.; Wang, X.; Gao, X.; He, X.; Zhao, W.; Lan, H.; Tian, J.; Yang, K.; Wang, S. Gas chromatography-mass spectrometry profiling of volatile compounds reveals metabolic changes in a non-aflatoxigenic Aspergillus flavus induced by 5-azacytidine. Toxins 2020, 12, 57. [CrossRef]

8. Tian, J.; Huang, B.; Luo, X.; Zeng, H.; Ban, X.; He, J.; Wang, Y. The control of Aspergillus flavus with Cinnamomum jensenianum Hand.-Mazz essential oil and its potential use as a food preservative. Food Chem. 2012, 130, 520-527. [CrossRef]

9. Shu, X.; Wang, Y.; Zhou, Q.; Li, M.; Hu, H.; Ma, Y.; Chen, X.; Ni, J.; Zhao, W.; Huang, S.; et al. Biological degradation of aflatoxin B(1) by cell-free extracts of Bacillus velezensis DY3108 with broad PH stability and excellent thermostability. Toxins 2018, 10, 330. [CrossRef] 
10. Alberts, J.F.; Engelbrecht, Y.; Steyn, P.S.; Holzapfel, W.H.; van Zyl, W.H. Biological degradation of aflatoxin B1 by Rhodococcus erythropolis cultures. Int. J. Food Microbiol. 2006, 109, 121-126. [CrossRef]

11. Teniola, O.D.; Addo, P.A.; Brost, I.M.; Farber, P.; Jany, K.D.; Alberts, J.F.; van Zyl, W.H.; Steyn, P.S.; Holzapfel, W.H. Degradation of aflatoxin B(1) by cell-free extracts of Rhodococcus erythropolis and Mycobacterium fluoranthenivorans sp. nov. DSM44556(T). Int. J. Food Microbiol. 2005, 105, 111-117. [CrossRef] [PubMed]

12. Ren, X.; Zhang, Q.; Zhang, W.; Mao, J.; Li, P. Control of aflatoxigenic molds by antagonistic microorganisms: Inhibitory behaviors, bioactive compounds, related mechanisms, and influencing factors. Toxins 2020, 12, 24. [CrossRef] [PubMed]

13. Damann, J. Atoxigenic Aspergillus flavus biological control of aflatoxin contamination: What is the mechanism? World Mycotoxin J. 2015, 8, 235-244. [CrossRef]

14. Shakeel, Q.; Lyu, A.; Zhang, J.; Wu, M.; Li, G.; Hsiang, T.; Yang, L. Biocontrol of Aspergillus flavus on peanut kernels using Streptomyces yanglinensis 3-10. Front. Microbiol. 2018, 9, 1049. [CrossRef]

15. Dorner, J.W.; Cole, R.J.; Blankenship, P.D. Use of a biocompetitive agent to control preharvest aflatoxin in drought stressed peanuts. J. Food Prot. 1992, 55, 888-892. [CrossRef]

16. Mann, R.; Rehm, H.-J. Degradation products from aflatoxin B1 by Corynebacterium rubrum, Aspergillus niger, Trichoderma viride and Mucor ambiguus. Eur. J. Appl. Microbiol. Biotechnol. 1976, 2, 297-306. [CrossRef]

17. Hua, S.S.T.; Sarreal, S.B.L.; Chang, P.K.; Yu, J. Transcriptional regulation of aflatoxin biosynthesis and conidiation in Aspergillus flavus by Wickerhamomyces anomalus WRL-076 for reduction of aflatoxin contamination. Toxins 2019, 11, 81. [CrossRef]

18. El Khoury, R.; Caceres, I.; Puel, O.; Bailly, S.; Atoui, A.; Oswald, I.P.; El Khoury, A.; Bailly, J.D. Identification of the anti-aflatoxinogenic activity of Micromeria graeca and elucidation of its molecular mechanism in Aspergillus flavus. Toxins 2017, 9, 87. [CrossRef]

19. Zhao, Y.; Zhang, C.; Folly, Y.M.E.; Chang, J.; Wang, Y.; Zhou, L.; Zhang, H.; Liu, Y. Morphological and transcriptomic analysis of the inhibitory effects of Lactobacillus plantarum on Aspergillus flavus Growth and Aflatoxin Production. Toxins 2019, 11, 636. [CrossRef]

20. Yao, G.; Yue, Y.; Fu, Y.; Fang, Z.; Xu, Z.; Ma, G.; Wang, S. Exploration of the regulatory mechanism of secondary metabolism by comparative transcriptomics in Aspergillus flavus. Front. Microbiol. 2018, 9, 1568. [CrossRef]

21. Cary, J.W.; Harris-Coward, P.Y.; Ehrlich, K.C.; Mack, B.M.; Kale, S.P.; Larey, C.; Calvo, A.M. NsdC and NsdD affect Aspergillus flavus morphogenesis and aflatoxin production. Eukaryot. Cell 2012, 11, 1104-1111. [CrossRef]

22. Luo, X.; Affeldt, K.J.; Keller, N.P. Characterization of the Far transcription factor family in Aspergillus flavus. G3 Genes Genomes Genet. 2016, 6, 3269-3281. [CrossRef] [PubMed]

23. Zhuang, Z.; Lohmar, J.M.; Satterlee, T.; Cary, J.W.; Calvo, A.M. The master transcription factor mtfA governs aflatoxin production, morphological development and pathogenicity in the fungus Aspergillus flavus. Toxins 2016, 8, 29. [CrossRef] [PubMed]

24. Wu, M.Y.; Mead, M.E.; Lee, M.K.; Ostrem Loss, E.M.; Kim, S.C.; Rokas, A.; Yu, J.H. Systematic dissection of the evolutionarily conserved WetA developmental regulator across a genus of filamentous fungi. mBio 2018, 9. [CrossRef] [PubMed]

25. Inglis, D.O.; Binkley, J.; Skrzypek, M.S.; Arnaud, M.B.; Cerqueira, G.C.; Shah, P.; Wymore, F.; Wortman, J.R.; Sherlock, G. Comprehensive annotation of secondary metabolite biosynthetic genes and gene clusters of Aspergillus nidulans, A. fumigatus, A. niger and A. oryzae. BMC Microbiol. 2013, 13, 91. [CrossRef]

26. Chen, C.; Chen, H.; He, Y.; Xia, R. TBtools, a Toolkit for Biologists integrating various biological data handling tools with a user-friendly interface. bioRxiv 2018, 289660. [CrossRef]

27. Drott, M.T.; Satterlee, T.R.; Skerker, J.M.; Pfannenstiel, B.T.; Glass, N.L.; Keller, N.P.; Milgroom, M.G. The Frequency of Sex: Population genomics reveals differences in recombination and population structure of the aflatoxin-producing fungus. mBio 2020, 11, e00963-20. [CrossRef]

28. Yan, S.; Liang, Y.; Zhang, J.; Liu, C.M. Aspergillus flavus grown in peptone as the carbon source exhibits spore density- and peptone concentration-dependent aflatoxin biosynthesis. BMC Microbiol. 2012, 12, 106. [CrossRef]

29. Amaike, S.; Keller, N.P. Aspergillus flavus. Annu. Rev. Phytopathol. 2011, 49, 107-133. [CrossRef]

30. Rudramurthy, S.M.; Seyedmousavi, S.; Dhaliwal, M.; Chakrabarti, A.; Meis, J.F.; Mouton, J.W. Pharmacodynamics of voriconazole against wild-type and azole-Resistant Aspergillus flavus isolates in a nonneutropenic murine model of disseminated aspergillosis. Antimicrob. Agents Chemother. 2017, 61. [CrossRef]

31. Zhang, T.; Shi, Z.-Q.; Hu, L.-B.; Cheng, L.-G.; Wang, F. Antifungal compounds from Bacillus subtilis B-FS06 inhibiting the growth of Aspergillus flavus. World J. Microbiol. Biotechnol. 2007, 24, 783-788. [CrossRef] 
32. Tian, J.; Wang, Y.; Lu, Z.; Sun, C.; Zhang, M.; Zhu, A.; Peng, X. Perillaldehyde, a promising antifungal agent used in food preservation, triggers apoptosis through a metacaspase-dependent pathway in Aspergillus flavus. J. Agric. Food Chem. 2016, 64, 7404-7413. [CrossRef]

33. Pan, C.; Li, Y.X.; Yang, K.; Famous, E.; Ma, Y.; He, X.; Geng, Q.; Liu, M.; Tian, J. The molecular mechanism of perillaldehyde inducing cell death in Aspergillus flavus by inhibiting energy metabolism revealed by transcriptome sequencing. Int. J. Mol. Sci. 2020, 21, 1518. [CrossRef]

34. Alshannaq, A.F.; Gibbons, J.G.; Lee, M.K.; Han, K.H.; Hong, S.B.; Yu, J.H. Controlling aflatoxin contamination and propagation of Aspergillus flavus by a soy-fermenting Aspergillus oryzae strain. Sci. Rep. 2018, 8, 16871. [CrossRef]

35. Xing, F.; Wang, L.; Liu, X.; Selvaraj, J.N.; Wang, Y.; Zhao, Y.; Liu, Y. Aflatoxin B1 inhibition in Aspergillus flavus by Aspergillus niger through downregulating expression of major biosynthetic genes and AFB1 degradation by atoxigenic A. flavus. Int. J. Food Microbiol. 2017, 256, 1-10. [CrossRef]

36. Xu, D.; Wang, H.; Zhang, Y.; Yang, Z.; Sun, X. Inhibition of non-toxigenic Aspergillus niger FS10 isolated from Chinese fermented soybean on growth and aflatoxin B1 production by Aspergillus flavus. Food Control 2013, 32, 359-365. [CrossRef]

37. Shantha, T.; Rati, E. Isolation and characterization of an aflatoxin-inhibiting metabolite from A. niger. Curr. Sci. 1990, 59, 326-327.

38. Delgado, J.; Owens, R.; Doyle, S.; Núñez, F.; Asensio, M. Quantitative proteomics reveals new insights into calcium-mediated resistance mechanisms in Aspergillus flavus against the antifungal protein PgAFP in cheese. Food Microbiol. 2017, 66, 1-10. [CrossRef]

39. Lee, D.G.; Shin, S.Y.; Maeng, C.-Y.; Jin, Z.Z.; Kim, K.L.; Hahm, K.-S. Isolation and characterization of a novel antifungal peptide from Aspergillus niger. Biochem. Biophys. Res. Commun. 1999, 263, 646-651.

40. Chen, Y.; Kong, Q.; Liang, Y. Three newly identified peptides from Bacillus megaterium strongly inhibit the growth and aflatoxin B 1 production of Aspergillus flavus. Food Control 2018, 21, 1518. [CrossRef]

41. Binder, U.; Oberparleiter, C.; Meyer, V.; Marx, F. The antifungal protein PAF interferes with PKC/MPK and cAMP/PKA signalling of Aspergillus nidulans. Mol. Microbiol. 2010, 75, 294-307. [CrossRef]

42. Raksha Rao, K.; Vipin, A.V.; Venkateswaran, G. Mechanism of inhibition of aflatoxin synthesis by non-aflatoxigenic strains of Aspergillus flavus. Microb. Pathog. 2020. [CrossRef]

43. Lind, A.L.; Lim, F.Y.; Soukup, A.A.; Keller, N.P.; Rokas, A. An LaeA- and BrlA-dependent cellular network governs tissue-specific secondary metabolism in the human pathogen Aspergillus fumigatus. mSphere 2018, 3. [CrossRef]

44. Yang, K.; Liu, Y.; Wang, S.; Wu, L.; Xie, R.; Lan, H.; Fasoyin, O.E.; Wang, Y.; Wang, S. Cyclase-associated protein Cap with multiple domains contributes to mycotoxin biosynthesis and fungal virulence in Aspergillus flavus. J. Agric. Food Chem. 2019, 67, 4200-4213. [CrossRef]

45. Kim, D.; Langmead, B.; Salzberg, S.L. HISAT: A fast spliced aligner with low memory requirements. Nat. Methods 2015, 12, 357-360. [CrossRef]

46. Li, H.; Handsaker, B.; Wysoker, A.; Fennell, T.; Ruan, J.; Homer, N.; Marth, G.; Abecasis, G.; Durbin, R. The sequence alignment/map format and SAMtools. Bioinformatics 2009, 25, 2078-2079. [CrossRef]

47. Liao, Y.; Smyth, G.K.; Shi, W. featureCounts: An efficient general purpose program for assigning sequence reads to genomic features. Bioinformatics 2014, 30, 923-930. [CrossRef]

48. Langfelder, P.; Horvath, S. WGCNA: An R package for weighted correlation network analysis. BMC Bioinform. 2008, 9, 559. [CrossRef]

(C) 2020 by the authors. Licensee MDPI, Basel, Switzerland. This article is an open access article distributed under the terms and conditions of the Creative Commons Attribution (CC BY) license (http://creativecommons.org/licenses/by/4.0/). 